
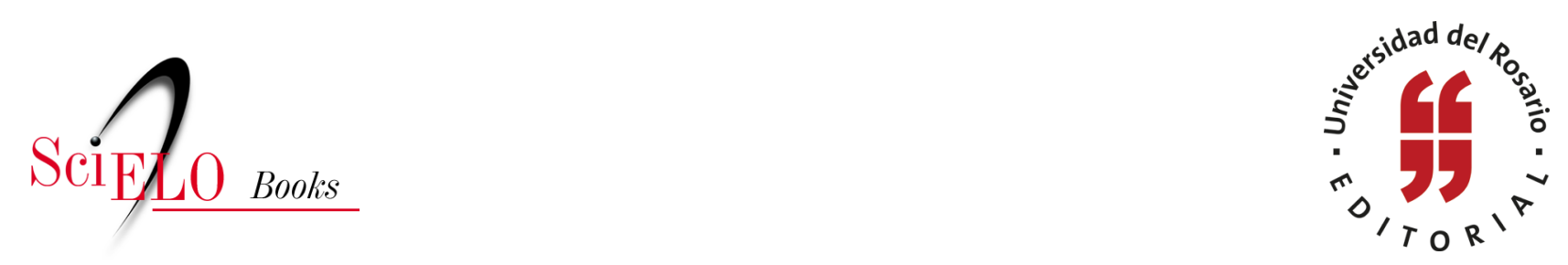

\title{
Capítulo 6. Alternativa de desmonte de la estratificación socioeconómica en el Distrito Capital
}

\author{
Juan Miguel Gallego \\ Luis H. Gutiérrez \\ Denis López \\ Carlos E. Sepúlveda
}

\section{SciELO Books / SciELO Livros / SciELO Libros}

GALLEGO, J.M., GUTIÉRREZ, L.H., LÓPEZ, D., and SEPÚLVEDA, C.E. Alternativa de desmonte de la estratificación socioeconómica en el Distrito Capital. In: SEPÚLVEDA RICO, C.E., LÓPEZ CAMACHO, D., and GALLEGO ACEVEDO, J.M., eds. Los límites de la estratificación: en busca de alternativas [online]. Bogotá: Editorial Universidad del Rosario: Alcaldía Mayor de Bogotá D.C., 2014, pp. 149-175. ISBN: 978-958-738-537-3. https://doi.org/10.7476/9789587385373.0008.

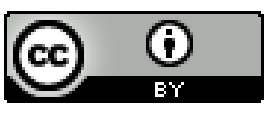

All the contents of this work, except where otherwise noted, is licensed under a Creative Commons Attribution 4.0 $\underline{\text { International license. }}$

Todo o conteúdo deste trabalho, exceto quando houver ressalva, é publicado sob a licença Creative Commons Atribição 4.0.

Todo el contenido de esta obra, excepto donde se indique lo contrario, está bajo licencia de la licencia $\underline{\text { Creative }}$ Commons Reconocimento 4.0. 


\title{
Capítulo 6 \\ Alternativa de desmonte de la estratificación socioeconómica en el Distrito Capital
}

\author{
Juan Miguel Gallego*, Luis H. Gutiérrez**, Denis López**** \\ y Carlos E. Sepúlveda***
}

Palabras clave: subsidios en servicios públicos domiciliarios, avalúo catastral, tarifas y subsidios, discriminación de precios, regulación en servicios públicos, equidistribución, progresividad.

\section{Introducción}

La estratificación socioeconómica, como herramienta de clasificación y agrupación de inmuebles residenciales, surgió con el propósito de facilitar la implementación de subsidios dentro del esquema de tarifas en servicios públicos domiciliarios (SPD), garantizando criterios de solidaridad, redistribución de ingresos, simplificación y transparencia. El objetivo inicial de este esquema de clasificación fue encontrar un diseño técnico que permitiera la expansión del acceso al servicio a niveles de cubrimiento universal y de garantizar el consumo básico de dichos servicios domiciliarios a todos los hogares más allá

\footnotetext{
* Profesor asociado, Facultad de Economía de la Universidad del Rosario.

** Profesor titular, Facultad de Economía de la Universidad del Rosario.

*** Consultor asociado Infométrika, SAS.

**** Profesor principal, Facultad de Economía de la Universidad del Rosario.
} 
de su capacidad de pago. Este tipo de esquemas, basados en estratificación de predios por grupos, es atípico en el contexto internacional, y, para niveles de cobertura cercanos al cubrimiento universal, puede tener problemas técnicos cuando los criterios de clasificación no se actualizan o no reflejan la realidad socioeconómica de los hogares.

La Ley 142 de 1994, como pilar normativo del esquema, planteó dos objetivos específicos. Primero, posibilitar el acceso de los SPD a toda la población a través de "(la) ampliación permanente de la cobertura mediante sistemas que compensen la insuficiencia de la capacidad de pago de los usuarios" (art. $\left.2^{\circ}\right)$; y, segundo, de redistribución y solidaridad, determinado en el artículo 87.3. "Por solidaridad y redistribución se entiende que al poner en práctica el régimen tarifario se adoptarán medidas para asignar recursos a 'fondos de solidaridad y redistribución', para que los usuarios de los estratos altos y los usuarios comerciales e industriales, ayuden a los usuarios de estratos bajos a pagar las tarifas de los servicios que cubran sus necesidades básicas"1.

El impacto de la política estatal sobre los servicios públicos domiciliarios fundamentado en la estratificación socioeconómica ciertamente logró de manera rápida un incremento de la cobertura casi universal en la mayoría del territorio nacional. En particular, esta cobertura alcanza cerca de un 100\% de energía eléctrica y acueducto para los hogares ubicados en zonas urbanas, jalonados principalmente por expansiones significativas en las grandes ciudades del país; y superior al $85 \%$ en las áreas rurales (Parra, 2011). Este crecimiento acelerado en cobertura permitió explotar las economías de escala que generan la conexión masiva de nuevos usuarios y afectó de manera favorable la fijación en el sistema.

Para el caso de Bogotá, D.C., la cobertura en acueducto, alcantarillado y aseo, energía eléctrica y gas llega a niveles cercanos al $100 \%$ en casi todos los servicios. Para el caso de gas, al tener un sustituto en energía eléctrica, su política de expansión de la red solo en años recientes ha alcanzado niveles de cobertura cercanos al $90 \%$ para Bogotá, según datos de la Encuesta multipropósito de Bogotá 2011 (EMB 2011).

1 La aplicación del esquema de tarifas y de subsidios estableció el siguiente sistema de subsidios y contribuciones (art. 99.6): Los hogares ubicados en estratos 1, 2 y 3 son receptores de subsidios de hasta el 50, 40 y $15 \%$, respectivamente; los hogares del estrato 4 cancelan el costo de prestación del servicio; y los hogares de los estratos 5 y 6 contribuyen pagando una tarifa un $20 \%$ superior a la tarifa de referencia del estrato 4 . 
Por otro lado, a pesar del avance en cobertura, la estratificación ha tenido un alcance mucho más limitado en sus objetivos de focalización y redistribución del ingreso. Diferentes estudios plantean que la estratificación, como mecanismo central en la definición de subsidios en servicios públicos domiciliarios: 1) genera altos errores de inclusión² (Vélez, 1996; Mina, 1997; CID-DAPD, 2004; Conpes 3386/05; Ramírez, 2007; Meléndez, 2004 y 2008; Econometría, 2006; DANE, 2011; Parra, 2011; López y Sepúlveda, cap. 5); 2) afecta de manera positiva a los hogares beneficiados pero no afecta la distribución del ingreso, son neutros en su progresividad, o regresivos (Sánchez y Núñez, 2000; Lasso, 2006; Parra, 2011; Gallego et al., 2014); 3) afecta la competitividad de los sectores industriales y comerciales (Santamaría et al., 2009); 4) termina siendo un mecanismo de exclusión o segregación (SDP, 2007; Uribe, 2008; Ríos, 2010).

Dentro de las alternativas de instrumentos para focalizar subsidios o definir contribuciones en SPD, los tres capítulos anteriores han construido los elementos necesarios para proponer al avalúo catastral como una fuente alternativa que nutra nuevos modelos de estratificación socioeconómica en el país y mejore los problemas evidenciados por la metodología actual. En el capítulo 5, López y Sepúlveda presentan modelos que resultan en una mejor clasificación en términos de que una menor proporción de hogares con altos ingresos habita viviendas clasificadas en estratos bajos, brindando una mejora notoria respecto a la sostenibilidad del sistema y a un mejor uso de los recursos públicos. Sin embargo, surgen varios dilemas. Un primer escenario que considera a toda la nación (y, por lo tanto, capta toda la diferenciación urbana del país) representa la alternativa que resulta en menores errores de inclusión, pero, a su vez, los cambios en los estratos asignados son tan drásticos para el Distrito Capital que, para hacer viable la implementación de esta opción, se requeriría de un largo período de transición que permita la aplicación gradual de los nuevos subsidios y contribuciones asociados a los nuevos grupos de clasificación, evitando generar fuertes incrementos en las facturas de servicios públicos de un mes de consumo a otro; además, de mantenerse los porcentajes de subsidios y contribuciones actuales, seguirían los problemas de progresividad. Los otros modelos toman a Bogotá de manera independiente del resto del país, y resultan en cambios menos fuertes y más factibles de implementar, pero los errores de

2 Hogares con alta capacidad de pago se ven beneficiados de subsidios. 
inclusión, a pesar de disminuir notablemente, siguen siendo significativos. La regresividad que tienen hoy los niveles de subsidios y contribuciones en cada grupo se mantendría y, en todo caso, se requerirá de un período de transición que reduzca el impacto en las facturas que trae los cambios en los nuevos niveles de subsidio o contribución al cambiar de estrato.

El hecho de que un eventual esfuerzo institucional para transitar a una nueva metodología de estratificación presente aún errores de inclusión significativos, independientemente del modelo utilizado, lleva a explorar alternativas que permitan incorporar en el diseño de esquemas tarifarios la información catastral como insumo en la clasificación de los inmuebles residenciales objeto de subsidios o contribuciones. Las ventajas que en su momento justificaron la estratificación pierden vigencia hoy en día ante la posibilidad de utilizar directamente el avalúo catastral para mejorar la focalización de subsidios cruzados de forma más detallada.

Este capítulo ilustra la manera como la información catastral podría usarse como base para focalizar los subsidios y contribuciones en SPD para Bogotá. En particular, se presenta un ejercicio que propone un escenario hipotético sin los estratos socioeconómicos y utiliza el avalúo catastral como mecanismo para determinar el monto de subsidio o contribución que recibiría el inmueble, como un vehículo de transmisión de subsidios o pago de contribuciones por parte de los miembros del hogar. Dicho ejercicio, aplicado al caso de acueducto, supone una elasticidad precio e ingreso en el consumo del servicio constante para todos los hogares, lo cual simplifica el diseño de tarifas con el fin de enfocar la propuesta en el poder discriminante del avalúo catastral para el diseño de subsidios y contribuciones en el pago del servicio ${ }^{3}$.

Este ejercicio basado en el avalúo, mostró mejoras significativas en la progresividad de los subsidios cruzados, gracias al poder discriminante de la información catastral con respecto a la capacidad de pago de los hogares. Aunque este escenario hipotético se aplicó al caso de acueducto, puede adaptarse a otro tipo de servicios públicos, como energía o gas. Lo importante de

3 Es importante anotar que esta propuesta no pretende sustituir los conceptos de diseños tarifarios que contienen incentivos al consumo eficiente por parte de los usuarios, y, por el contrario, se complementa con la regulación al consumo contenida en los diseños tarifarios actuales. Es necesario incluir en la agenda futura el diseño de propuestas que complementen el componente de eficiencia y distribución basado en el avalúo, y depurar el ejercicio de este capítulo de manera que no resulte en un subsidio a los consumos totales de SPD. 
resaltar es el potencial discriminador y la facilidad de aplicar la información catastral para focalizar subsidios y contribuciones, y mejorar el componente distributivo de un esquema tarifario, lo cual puede ser compatible con los incentivos diseñados para el consumo eficiente del servicio.

El capítulo analiza algunas experiencias internacionales en términos de diseño de subsidios y tarifas, y en mecanismos de focalización, particularmente para el caso de América Latina (sección 6.1). Posteriormente, compara las principales características de las experiencias internacionales expresadas en la sección anterior con el sistema de estratificación colombiano (sección 6.2). La sección 6.3 introduce el ejercicio de desmonte de la estratificación determinando los subsidios y contribuciones directamente del avalúo catastral. La sección 6.4 describe las bases de datos utilizadas y los pasos metodológicos. La sección 6.5 presenta los resultados del ejercicio, y, finalmente, se ofrecen unas conclusiones.

\subsection{Implantación de tarifas y subsidios a servicios públicos domiciliarios en la práctica}

A nivel internacional, tanto en países desarrollados como en desarrollo se ha considerado el acceso y uso a los servicios públicos domiciliarios de parte de todos los habitantes como una obligación del Estado. La aplicación de los esquemas tarifarios y de las formas de aplicación de subsidios focalizados varía de un país a otro. Esta sección presenta de manera general algunas experiencias internacionales, especialmente en América Latina, respecto a la implantación de subsidios dirigidos a los usuarios de servicios públicos domiciliarios.

La forma de abordar el diseño de subsidios a usuarios de SPD abarca dos aspectos principales. Por un lado, el diseño de tarifas diferenciales (focalización por cantidades consumidas) y, por el otro, el modo de identificar a los beneficiarios de la política de subsidios (selección administrativa). La tabla 6.1 presenta algunos ejemplos de fijación de tarifas y de subsidios para el sector de agua potable en países de América Latina. Una descripción más completa sobre esquemas de tarificación en varios SPD se encuentra en Komives et al. (2005). De otra parte, el informe del IMF (2013) presenta la evolución de tarifas y subsidios a la energía eléctrica para un grupo de ocho países en desarrollo. Adicionalmente, Briceño y Shkaratan (2011) realizan un exhaustivo y más reciente análisis de las tarifas y subsidios para los servicios públicos en 25 países africanos. 
Para el caso de agua potable en América Latina, un buen número de países ha adoptado esquemas de tarifas por bloques crecientes con adición de cargos fijos (con el objetivo de cubrir costos fijos o de inversión para asegurar la sostenibilidad en la prestación). También, han predominado los esquemas de subsidios cruzados donde algunos sectores financian mediante tarifas más altas a los hogares de menores ingresos (tabla 6.1).

\section{Tabla 6.1. Algunas experiencias de fijación de tarifas y subsidios en América Latina}

\begin{tabular}{|c|c|c|c|}
\hline País & Existencia de subsidios o no & Focalización & Cargos fijos \\
\hline Argentina & $\begin{array}{l}\text { Varios tipos de subsidios. Al consumo, mediante la llamada } \\
\text { tarifa social, consistente en un descuento de } 4 \text { pesos argentinos } \\
\text { más el IVA sujeto a comprobación previa de medios de vida } \\
\text { (CPMV). Cruzados al consumo sobre base geográfica; y cruzados } \\
\text { al consumo sobre base de proxies de capacidad de pago como, } \\
\text { por ejemplo, factores de calidad y antigüedad de la vivienda } \\
\text { (factores } Z \text { y E). Es más alto cuanto mayor es la calidad y menor } \\
\text { respecto a la antigüedad. }\end{array}$ & $\begin{array}{l}\text { Aparentemente } \\
\text { el esquema } \\
\text { de susbidio es } \\
\text { universal y no } \\
\text { focalizado. }\end{array}$ & $\begin{array}{l}\text { Existen cargos fijos } \\
\text { por volumenes. } \\
\text { Para residenciales } \\
\text { con medidor, } \\
\text { este es de } 20 \\
\text { metros cúbicos } \\
\text { bimestrales. }\end{array}$ \\
\hline Brasil & $\begin{array}{l}\text { Esquemas diferentes según la empresa. } 1 \text {. Subsidios al consumo } \\
\text { que cubren de manera temporal, en forma parcial o total el } \\
\text { pago de la tarifa. Para las categorías normal y social y favelas } \\
\text { (CPMV). } 2 \text {. Subsidios cruzados al consumo a partir de categorías } \\
\text { de clientes. Los no residenciales subsidian a los residenciales } \\
\text { y varios de estos últimos subsidian a las categorías normal, } \\
\text { social y favelas. } 3 \text {. Subsidios cruzados al consumo a partir de } \\
\text { categorías de clientes. La tarifa social es del } 60 \% \text { de la normal } \\
\text { residencial para los primeros } 6 \text { metros cúbicos; } 80 \% \text { entre } 6 \text { y } \\
10 \text { m cúbicos, y } 90 \% \text { entre } 10 \text { y } 20 \text { m cúbicos. }\end{array}$ & $\begin{array}{l}\text { El esquema es } \\
\text { más focalizado. }\end{array}$ & $\begin{array}{l}\text { No parecen existir } \\
\text { cargos fijos. }\end{array}$ \\
\hline Chile & $\begin{array}{l}\text { Subsidios directos al consumo. Descuentos por los primeros } 15 \\
\text { m cúbicos que fluctúan entre el } 25 \% \text { - } 85 \% \text {. Los hogares en el } \\
\text { Sistema de Protección Social Chile Solidario reciben el } 100 \% \\
\text { de la cuenta hasta los primeros } 15 \text { m cúbicos. }\end{array}$ & $\begin{array}{l}\text { El esquema es } \\
\text { focalizado. }\end{array}$ & $\begin{array}{l}\text { No parecen existir } \\
\text { cargos fijos. }\end{array}$ \\
\hline $\begin{array}{l}\text { Costa } \\
\text { Rica }\end{array}$ & $\begin{array}{l}\text { Hay dos estructuras. Para clientes con y sin medición. Para } \\
\text { los que tienen medición, hay estructuras de tarifas en bloque } \\
\text { crecientes. Para los que no tienen medición, se aplica una } \\
\text { tarifa plana. }\end{array}$ & & $\begin{array}{l}\text { Para los que tienen } \\
\text { medidor, hay un } \\
\text { cargo fijo uniforme } \\
\text { independiente del } \\
\text { consumo. }\end{array}$ \\
\hline Ecuador & $\begin{array}{l}\text { Subsidios cruzados por estratificación socioeconómica de } \\
\text { los residenciales, siendo lo más cercano al caso colombiano. } \\
\text { Existe una tarifa social para usuarios en los sectores } 9,8,7,6 \\
\text { y } 5 \text {, definidos como sector económico bajo, bajo medio, bajo } \\
\text { alto, medio bajo y medio, respectivamente, con descuentos } \\
\text { del } 22 \% \text { para los sectores } 9 \text { y } 8,10 \% \text { para los sectores } 5 \text { y } 6, \text { y } \\
5 \% \text { para el sector } 5 \text {. }\end{array}$ & $\begin{array}{l}\text { El esquema es } \\
\text { más focalizado. }\end{array}$ & $\begin{array}{l}\text { Hay un cargo } \\
\text { fijo uniforme } \\
\text { independiente del } \\
\text { consumo. }\end{array}$ \\
\hline
\end{tabular}




\begin{tabular}{|c|c|c|c|}
\hline País & Existencia de subsidios o no & Focalización & Cargos fijos \\
\hline Perú & $\begin{array}{l}\text { Varias categorías de usuarios. En los residenciales, dos: los } \\
\text { llamados sociales y los domésticos. La tarifa social es menor } \\
\text { que la tarifa correspondiente al primer rango de la categoría } \\
\text { doméstica. Las categorías domésticas (3) van por rangos de } \\
\text { consumo con tarifas crecientes. En general, la regla es que } \\
\text { "Tarifa social es menor que tarifa correspondiente al primer } \\
\text { rango de la categoría doméstica, que a su vez es menor que tarifa } \\
\text { del segundo rango de la categoría doméstica, que es menor que } \\
\text { tarifa del tercer rango de la categoría doméstica, que es menor } \\
\text { que tarifa de la categoría estatal, que es menor que tarifa de } \\
\text { la categoría comercial, que a su vez es menor que tarifa de la } \\
\text { categoría industrial". }\end{array}$ & $\begin{array}{l}\text { El esquema es } \\
\text { focalizado vía } \\
\text { el Sistema de } \\
\text { Focalización } \\
\text { de Hogares } \\
\text { (SISFOH). }\end{array}$ & Hay cargos fijos. \\
\hline Panamá & $\begin{array}{l}\text { Subsidios cruzados geográficos. Los habitantes de Panamá y } \\
\text { Colón tienen tarifas superiores a las del interior. Las tarifas son } \\
\text { en bloques de consumo y crecientes. Los sectores comerciales } \\
\text { e industriales financian a los residenciales hasta los primeros } \\
15 \text { m cúbicos. }\end{array}$ & & \\
\hline Paraguay & $\begin{array}{l}\text { Tres categorías de usuarios. Residencial subsidiado, y residencial } \\
\text { no subsidiado y no residencial. Tarifas en bloques para los tres } \\
\text { grupos pero con tarifas inferiores a cada bloque de consumo } \\
\text { para los residenciales subsidiados. }\end{array}$ & & $\begin{array}{l}\text { Hay cargos fijos } \\
\text { diferenciados por } \\
\text { si el hogar recibe o } \\
\text { no subsidios. }\end{array}$ \\
\hline Uruguay & $\begin{array}{l}\text { Se subsidian hasta } 15 \mathrm{~m} \text { cúbicos los cargos fijos y variables } \\
\text { en los casos de planes sociales, inmuebles en asentamientos } \\
\text { (identificados por el Ministerio de Vivienda) y hogares en } \\
\text { condiciones de vulnerabilidad (clasificados por la Dirección } \\
\text { Nacional de Vivienda). }\end{array}$ & & $\begin{array}{l}\text { Cargo fijo variable } \\
\text { por tipo de cliente. }\end{array}$ \\
\hline
\end{tabular}

Fuente: Ferro y Lentini (2013).

La revisión de la literatura internacional sobre análisis de tarifas y esquemas de subsidios muestra algunos puntos comunes que vale la pena recalcar. El primero es que la mayoría de los países ha adoptado básicamente estructuras tarifarias no lineales, más precisamente tarifas de bloques crecientes (TBC) ${ }^{4}, \mathrm{o}$ tarifas en dos partes, y en menor porcentaje tarifas lineales. Briceño y Shkaratan (2011) encontraron que dos tercios de los países africanos en los que estudiaron las tarifas eléctricas residenciales usaron TBC, y el restante tarifas lineales. Ferro y Lentini (2013), en su estudio del sector de agua potable para algunos países de América Latina, destacan, igualmente, el predominio de las tarifas no lineales, incluso con esquemas complejos, en línea con el estudio clásico de Komives et al. (2005). Los esquemas de tarifas en bloque creciente

4 TBC es una tarifa escalonada que cobra un precio mayor para bloques de consumo crecientes. 
son también mayoritarios en la tarificación en los Estados Unidos (Borenstein, 2012). El uso de ese tipo de tarificación se ha justificado por factores de eficiencia, es decir, permitir que la empresa proveedora del servicio pueda recuperar costos. Los esquemas de subsidios se han anexado a las tarifas en dos partes o a las tarifas en bloque. En esos esquemas, llamados algunas veces tarifas sociales, se otorgan o consumos mínimos vitales totalmente subsidiados, o estos consumos se otorgan con altos descuentos respecto al costo marginal de provisión del servicio.

En segundo lugar, el aspecto central más problemático en el diseño del otorgamiento de subsidios es el targeting o elección del sujeto beneficiario del subsidio. Dentro de las experiencias analizadas, un primer grupo de países otorga subsidios de acuerdo con criterios geográficos; es decir, zonas urbanas marginales (áreas geográficas o barrios) que acogen a la mayoría de la población pobre. La manera de financiar estos esquemas es amplia: presupuesto nacional directo, subsidios cruzados por zonas geográficas (distinciones entre zonas urbanas y rurales, o por tipos de ciudad, para montar los subsidios cruzados o, en su defecto, transferencias gubernamentales a los proveedores).

Otro grupo de países ha construido sistemas que focalizan subsidios de SPD directamente en la unidad de gasto (hogares) o individuos, a través de selección administrativa y métodos multivariados que requieren comprobación de condiciones de vida a través de múltiples factores asociados al ingreso (Komives et al., 2005). Los casos de elección de potenciales beneficiarios en Chile para el servicio de agua potable a través de la ficha CAS (comités de asistencia social), o el Sistema de Focalización de Hogares (Sisfoh) en Perú, o el programa CARE en California para energía eléctrica (California Alternate Rates for Energy) identifican, mediante registros administrativos de hogares o empadronamientos socioeconómicos, los eventuales beneficiarios de subsidios directos o de tarifas diferenciales bajas en SPD. Estos esquemas pretenden identificar hogares de menores ingresos y su disposición de pago. Indirectamente, la asignación de subsidios en esos casos podría corresponder o acercarse a una forma de discriminación perfecta de otorgar subsidios.

Dos observaciones vale la pena señalar respecto a estas experiencias internacionales. De una parte, al tener que informar los solicitantes sus niveles de ingreso, hacen del esquema de subsidio un esquema más progresivo y transparente en el tiempo, dado que, en la medida en que los niveles educativos y de ingreso de las personas se mejoren (o empeoren), puedan dejar de ser (o 
llegar a serlo en caso de caída en los ingresos) beneficiarias del subsidio. Sin embargo, estas experiencias deben fundamentarse en contextos institucionales de permanente provisión, manejo de información y actualización, a partir de procesos exigentes, transparentes y libres de influencias políticas.

En Colombia, se estableció desde el año 1994 un mecanismo de identificación conocido como el sISBEN o Sistema de Identificación de Potenciales Beneficiarios para Programas Sociales, el cual ha buscado identificar a la población pobre del país mediante la cuantificación de las condiciones socioeconómicas de los individuos ${ }^{5}$. Un registro de este tipo podría considerarse como una opción de focalización para la entrega de los subsidios a los SPD a la población pobre del país. Sin embargo, un reciente estudio que analiza la evolución del SISBEN1 al SISBEN2 concluye: "La evidencia encontrada sugiere que los hogares en condiciones relativamente mejores se benefician más de la respuesta estratégica, haciendo de ella una herramienta regresiva. En efecto, cuando los hogares son clasificados con base al puntaje del nuevo sIsBEN, cerca de una quinta parte de los beneficiarios del régimen subsidiado de salud lograrían ser incluidos gracias a la respuesta estratégica" (Bottia, Cardona y Medina, 2012, p. 165). Adicionalmente, registros de beneficiarios de programas sociales no permitirían un esquema cruzado de subsidios, ventaja del sistema colombiano actual.

\subsection{La política tarifaria implícita de la estratificación socioeconómica de la Ley 142 de 1994}

En la exposición de motivos de la Ley 142 de 1994, se reconocía que los segmentos más pobres de la población no podían cubrir los costos de prestación de los servicios públicos domiciliarios, razón por la cual se requería establecer un mecanismo de asignación de subsidios que evitara en lo posible que fuera regresivo en el sentido de favorecer a los grupos de mayores ingresos. La exposición de motivos señalaba la necesidad de usar "la estratificación socioeconómica de los usuarios con el fin de identificar a la población de escasos recursos, susceptibles de recibir subsidios para pago de servicios". La existencia y previa experiencia en el país de la estratificación socioeconómica sirvió entonces para garantizar (o posibilitar el cumplimiento) no solo del

\footnotetext{
"El SISBEN es el principal instrumento de focalización individual. Al menos 8 instituciones y 31 programas lo utilizan actualmente como criterio principal o complementario" (Conpes Social 117/08).
} 
mandato constitucional del derecho de los colombianos a los SPD, sino también para estructurar un esquema único de subsidios que, en teoría, garantizara la sostenibilidad del sistema general de tarifas y subsidios y la solidaridad.

Según el servicio público domiciliario, se establecieron unos porcentajes de deducción sobre el costo de prestación del servicio (o tarifa cobrada al estrato 4) para los estratos 1, 2 y 3 sobre consumos de subsistencia y porcentajes de contribuciones sobre ese costo a los estratos 5 y 6 (la figura 6.1 presenta el esquema que refleja la fijación de precios de acueducto por estrato socioeconómico $\left.{ }^{6}\right)$. La Ley 142 de 1994 estableció que, de existir déficits entre los montos de contribuciones y los de subsidios, estos debían ser cubiertos por los gobiernos locales o, en su defecto, por recursos del presupuesto nacional.

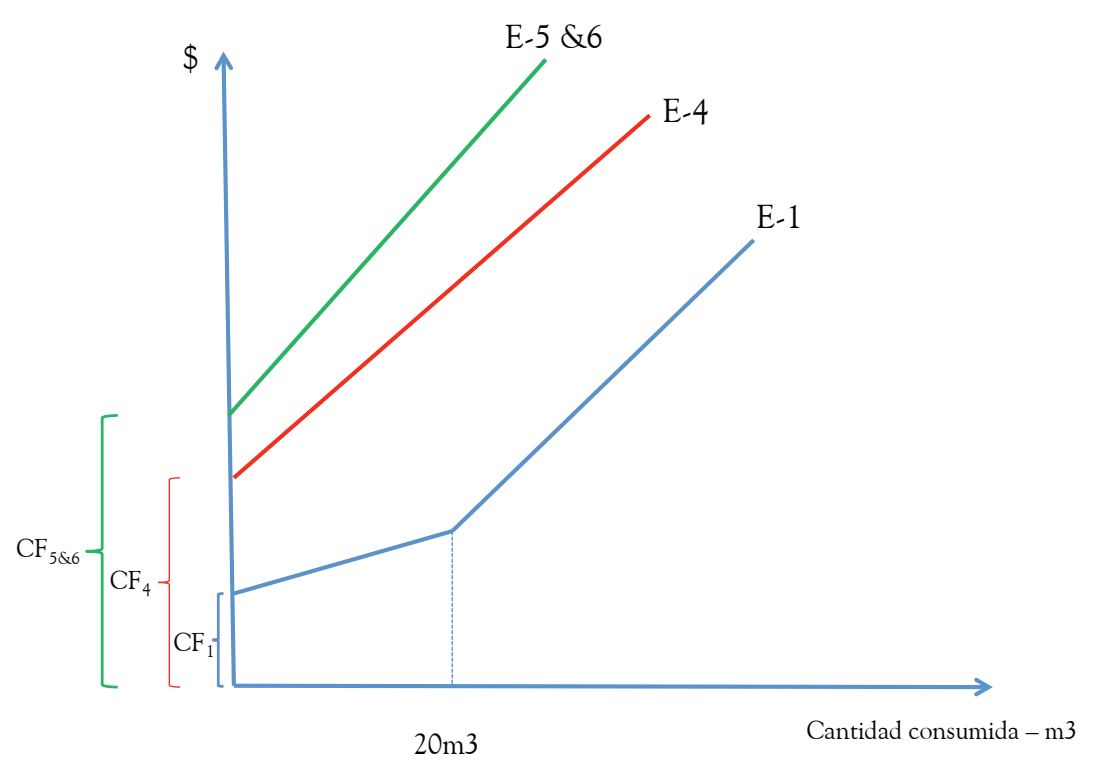

Figura 6.1. Esquema de las tarifas de agua derivadas de la estratificación de la Ley 142 de 1994

Fuente: elaboración de los autores.

6 El esquema se realiza con base en la información del documento Costos y tarifas. Municipios menores y zonas rurales del Ministerio de Ambiente, Vivienda y Desarrollo Social y la Usaid, 2005. No introduce el esquema ninguna de las actuales disposiciones sobre el consumo mínimo vital de $6 \mathrm{~m}^{3}$ de agua vigentes en Bogotá desde 2013. El consumo básico de subsistencia se toma del señalado por el Conpes 3386 de 2005. Por simplicidad, se dejaron de lado las tarifas a los estratos 2 y 3. 
Al estar fundamentado el esquema de subsidios en un sistema de estratificación basado en las características del inmueble que habita el hogar, el caso colombiano de asignación de subsidios a los SPD es (casi) único a nivel mundial, aunque califica como un esquema de subsidios cruzados. Como se indicaba ya en la exposición de motivos de la que sería la Ley 142 de 1994, la estratificación buscaba ser el mecanismo de 'seleccionar' a los hogares de menores ingresos susceptibles de ser beneficiarios del subsidio. Esto resulta en un esquema intermedio entre los criterios de selección administrativa geográficamente (vivienda) y por verificación de condiciones de vida (vivienda como proxy de las condiciones socioeconómicas del hogar). La experiencia internacional no muestra, a excepción del caso de Quito en Ecuador, un esquema de subsidios cruzados basados en estratos socioeconómicos como el colombiano (Ferro y Lentini, 2013).

¿Cómo se puede relacionar la tarificación derivada de la estratificación socioeconómica de la Ley 142 con los aportes teóricos económicos y las experiencias internacionales sobre el tema? En primer lugar, la estratificación implícitamente segmentó el mercado en seis estratos, lo cual clasificó a los usuarios por (potencialmente) distintos niveles de ingresos y, de alguna manera, el esquema capturaba diferencias sea en demanda o en las disposiciones por pagar por incrementos en consumo de parte de los hogares de los diversos estratos. Al clasificar los inmuebles de los hogares por grupos y establecer tarifas iguales en el interior de ellos, pero diferentes entre grupos, la tarificación implícitamente es cercana a la discriminación de precios de tercer grado. El esquema liberó a las empresas de buscar señales sobre las disposiciones por pagar de los hogares. El esquema de precios derivado de la estratificación adopta de los precios no lineales las tarifas en dos partes. Se observa que a todos los estratos se les cobra un cargo fijo (CF en la figura). En el caso colombiano el cargo fijo cubre costos de administración, operación y mantenimiento (AOM) que legalmente no pueden ser subsidiables, y, parcialmente, costos de inversión. El CF del estrato 4 cubre componentes del costo medio que no son subsidiables, y los estratos 1 a 3 pagan a través del CF y de una tarifa por consumo, un valor que es menor al costo medio. Por ejemplo, para el estrato 1, las tarifas incorporan subsidios hasta del $70 \%$ (en la figura 6.1), mientras que los estratos 5 y 6 pagan un sobreprecio del $20 \%$ sobre el CMA del estrato 4 .

Como lo observa Wilson (1993, p. 9), las tarifas no lineales y, más precisamente, la discriminación de precios pueden tener efectos distributivos 
adversos y promover usos anticompetitivos del poder de monopolio. La Ley 142, usando la estratificación como ancla, incorporó los elementos de subsidios para contrarrestar esos potenciales efectos negativos distributivos. Los efectos negativos en este caso pueden ser la exclusión de consumidores de bajos ingresos del mercado del servicio público domiciliario, lo cual contravendría los objetivos de la Ley 142 de ampliar la cobertura y mejorar el bienestar social y económico. Se observa en la figura 6.1 que por unidad de consumo, representada en el eje horizontal, un hogar en estrato 1 paga una tarifa inferior en un $70 \%$ sobre el costo medio de largo plazo $\left(\mathrm{CM}_{\mathrm{LP}}\right)$ de proveer el servicio, que es la tarifa pagada por el estrato 4 , y los estratos 5 y 6 contribuyen a financiar los subsidios pagando una tarifa superior en un $20 \%$ al costo de provisión del servicio.

¿Es eficiente el esquema de tarificación que se derivó a partir de la expedición de la Ley 142 y de la estratificación socioeconómica allí establecida? Varias observaciones, mas no respuestas, se podrían aventurar. La primera es que uno de los objetivos de la estratificación se ha logrado en la práctica. Este es el aumento de la cobertura, la cual llega al 100\% en las principales ciudades del país. La segunda es que el esquema ha llevado a una redistribución negativa del ingreso, tal como lo han cuantificado analistas del tema por los múltiples errores de inclusión y exclusión. La tercera es que el desarrollo de sistemas de información alternativos de focalización de quienes podrían ser beneficiarios de subsidios para consumir los servicios ha avanzado, lo cual mejoraría el bienestar de todos los grupos de la población, aliviando las finanzas públicas. Un ejemplo de esos sistemas se considera en la siguiente sección. Y una cuarta es que el sistema es pobremente redistributivo (incluso regresivo), pues exige a aquellos hogares de menor capacidad económica una mayor proporción de sus ingresos para pagar los SPD que a aquellos hogares de mayor capacidad económica.

\subsection{Alternativa granular a la estratificación socioeconómica en el Distrito Capital}

Los avances en cobertura en la prestación de los SPD, en particular en Bogotá, fueron posibles gracias al esquema cruzado de subsidios que se operacionalizó con la estratificación socioeconómica. La búsqueda de alternativas para hacer más eficiente el esquema de subsidios cruzados pasa por explorar 
alternativas que hagan tan granular la clasificación de domicilios que no implique su agrupación en un número definido de estratos. Es posible pensar estas opciones basados en sistemas alternativos de identificación que se aproximen a las disposiciones por pagar de los hogares y permitan sustituir el uso de la estratificación como mecanismo de asignación de los subsidios. En el capítulo 4, los autores identifican altas correlaciones del avalúo catastral y otras variables disponibles en el catastro de Bogotá, con múltiples indicadores socioeconómicos de los hogares. Si el avalúo es un buen predictor de los ingresos de los hogares, puede utilizarse como mecanismo alternativo que haga posible la operacionalización del sistema cruzado de subsidios y que sirva a los reguladores para incorporar esquemas alternativos de tarificación y de asignación de subsidios de SPD a los hogares pobres de la ciudad.

En línea con lo planteado por González (2013), dado que la información catastral brinda la caracterización más objetiva disponible sobre los bienes inmuebles residenciales, en caso de no existir una evidencia clara acerca de la utilidad de tener estos grupos diferenciados, sería más pertinente establecer un sistema de subsidios y contribuciones directamente a partir del avalúo catastral. Adicionalmente, llevaría a un redireccionamiento de recursos públicos al desmontar un proceso de recolección de información paralelo y que no complementa necesariamente lo que el catastro ofrece. Esto sin tener en cuenta consecuencias adicionales negativas que puede llegar a tener la estratificación y que este libro no aborda (segregación, estigmatización de pobres, incentivos negativos en agentes de mercado, entre otros).

A continuación, se expone un primer ejercicio exploratorio para Bogotá, D.C., que establece subsidios y contribuciones de tarifas de SPD, sin utilizar el esquema de estratos socioeconómicos o clasificación en grupos de hogares (en predios residenciales o viviendas) y que resulte en un esquema de pago de servicio público domiciliario ligado a información asociada al respectivo avalúo catastral de los bienes inmuebles.

Partiendo de Acosta et al. (cap. 4), se toma la variable de valor unitario integral del avalúo (VUI) como aquella que recoge adecuadamente la información de precios de los inmuebles residenciales y que, a su vez, está estrechamente ligada a la capacidad de pago de los hogares. El vUI homologa el valor del metro cuadrado de los predios de propiedad horizontal ( $\mathrm{PH}$ ) y los predios en no PH (en principio, el valor directo de la construcción no es comparable 
debido a las diferencias en los tamaños del terreno). El valor unitario integral del avalúo se define de la siguiente manera:

Valor unitario integral $= \begin{cases}\frac{\text { Avalúo }}{\text { área construida }} & \text { si es PH } \\ \text { Valor M2 Terreno }+ \text { Valor M2ConstPonderado } & \text { si es NPH }\end{cases}$

Donde ValorM2ConstPonderado corresponde a un promedio del valor de la construcción de las unidades con uso residencial ponderado por el área de construcción respectiva a cada unidad.

Este escenario hipotético de subsidios y contribuciones se aplica al caso de acueducto, pero este mismo ejercicio puede aplicarse a los otros tipos de servicios públicos, como energía o gas. Lo importante de resaltar con este ejercicio es que muestra el potencial discriminador y la facilidad de aplicar la información catastral construyendo una variable como el vUI del para focalizar los subsidios y contribuciones y mejorar el componente distributivo de un esquema tarifario.

El trabajo parte de dos principios. Siguiendo el propósito de redistribución definido por la Ley 142 de 1994, se construye un escenario alrededor de la definición de subsidios y contribuciones en SPD cuyas facturas reflejen un 'equiesfuerzo' en el gasto en acueducto como porcentaje del gasto total del hogar. Esto es que el esquema de subsidios y contribuciones cruzadas, aplicado a través de los inmuebles, resulte no regresivo, de forma que en promedio la proporción de pago del servicio de acueducto (o en general de SPD) de los hogares con relación al total del gasto (como proxy del ingreso) sea semejante y no decreciente en el ingreso como es el caso bajo el sistema de estratificación actual.

Es decir que el gasto en el servicio representará una misma proporción del gasto total para todos los hogares bogotanos. Se hace esta propuesta de 'equiesfuerzo' buscando garantizar un sistema cruzado, no regresivo (neutral en el ingreso) en la factura (pago) por unidad consumida, pero sin resultar en variaciones extremas en el pago para hogares contribuyentes con respecto a los pagos actuales bajo el esquema de estratificación. Como segundo principio, el ejercicio se ha concebido buscando mantener el mismo cierre financiero para la empresa de servicios públicos domiciliarios (ESP), garantizando una sostenibilidad de la propuesta. 
La aplicación del primer principio parte del supuesto que la utilidad de los hogares en el consumo de SPD exhibe una elasticidad precio e ingreso constante, este supuesto nos permite simplificar los efectos del precio y del ingreso sobre el consumo de los hogares y de este modo proponer una tarifa "equiesfuerzo" donde la proporción del gasto en SPD como proporción del gasto total (proxy del ingreso) es igual para todos los hogares. Esta simplificación es útil para poder resaltar el poder discriminatorio de nuestra propuesta de avalúo catastral como mecanismo para clasificar hogares receptores de subsidios u hogares contribuyentes manteniendo constante los efectos sobre el consumo de cambios en el valor a pagar?

\subsection{Metodología y bases de datos}

En esta sección, se describen los pasos establecidos y la base de datos utilizada para proponer un esquema de subsidios de tarifas en el que cada hogar haga un mismo 'esfuerzo' (factura de equiesfuerzo), definido como el porcentaje del gasto en acueducto respecto al gasto total, teniendo, en el total, los mismos ingresos (facturación) de las empresas que facturan, lo que mantiene intacta la sostenibilidad fiscal del sistema ${ }^{8}$.

\subsubsection{BASE DE DATOS}

Se utilizan tres fuentes principales de información: 1) la Encuesta multipropósito de Bogotá 2011 (EMB 2011), la cual nos permite construir la estructura de gasto y consumo de los hogares en acueducto, y en general su patrón de gasto; 2) información de las facturas de la empresas de servicios públicos (ESP) que es

7 Es importante anotar que este ejercicio se realiza manteniendo el mismo nivel de consumo del servicio por cada uno de los hogares, lo cual no necesariamente ocurre si los hogares, como se esperaría que ocurra, responden en consumo al gasto percibido. Un ejercicio más completo requiere una estimación de las elasticidades precio (en este caso facturación) del consumo del servicio a través de un sistema lineal de gasto, pero, dada la naturaleza descriptiva que quiere hacer el ejercicio propuesto de la capacidad redistributiva de la identificación de los predios y su relación con la capacidad de pago de los hogares, es suficiente con dejar de lado los efectos de incentivos económicos que generar los cambios en la factura sobre el comportamiento en consumo de los hogares. Una implementación de la propuesta de facturación de SPD a través de información catastral en lugar de un esquema de estratificación requiere de un análisis riguroso de los efectos de los cambios tarifarios sobre el consumo.

8 No se revisa en este ejercicio el impacto sobre la cartera, pero se puede suponer que, al ser más progresiva la tarifa que resulta de un 'equiesfuerzo', se podrá mejorar la cartera toda vez que la carga en servicios públicos disminuye para los hogares más vulnerables. 
reportada a través del Sistema Único de Información de Servicios Públicos (SUI) de la Superintendencia de Servicios Públicos (SUI $)^{9}$; 3 ) información sobre vigencia catastral 2012, la cual nos permite construir el vUI. Con el objetivo de poder calcular la participación del gasto en acueducto sobre el total de gasto para cada hogar, debe armonizarse primero la información de la EMB 2011 con la información de las facturas de las empresas de servicios públicos que es reportada a través del Sistema Único de Información.

En el proceso de emparejamiento entre las bases, se utilizó como variable de enlace el código catastral o CHIP adjudicado al predio al cual pertenece el hogar. Este proceso permite asociar la información de los hogares contenida en la EMB 2011 con los datos en catastro y en el SUI. Es importante resaltar que alguna información se pierde en este proceso, pues los CHIP de los hogares que se incluyeron en la EMB 2011 no coinciden con algún CHIP de la información catastral o no pueden ser emparejados con el CHIP disponible en la base del SUI. Inicialmente existen 16.486 hogares en la EMB 2011, de los cuales el 99,6\% (16.419) cuenta con la información de predio o CHIP al que pertenecen. Al cruzar con la base del SUI no fue posible ubicar el CHIP para 2.180 hogares, por lo que la base final de hogares resulta en el 86,8\% (12.280) de los hogares de la EMB $2011^{10}$.

Una vez identificado a través del CHIP cada predio y hogar de la EMB 2011, dentro del reporte del sur se puede obtener el costo de la prestación del servicio, establecido como la factura de consumo descontando los subsidios y las contribuciones, toda esta información proveniente del SUI. Para aquellos hogares con varias facturas, se suma el valor facturado por consumo para todas las facturas del predio y se asigna a cada hogar un valor de facturación por consumo ponderado por el número de personas que componen el hogar.

El proceso de emparejamiento de las bases de datos no garantiza que el costo de prestación de servicios por estratos en Bogotá reportado por la ESP por medio del sui se ajuste financieramente con el costo de la prestación de servicios que se puede obtener al expandir la muestra de los 12.280 hogares

9 Solo se realiza el ejercicio con el servicio de acueducto, dado que la información de las ESP únicamente tiene asociado el chIP para los servicios de acueducto, alcantarillado y aseo.

$10 \mathrm{Al}$ analizar por decil de capacidad de pago los promedios de facturación entre los hogares que quedaron en la muestra y aquellos que no, los promedios de los dos grupos no son significativamente diferentes. Los p-valores oscilan entre 0,11 y 0,87 . 
de la ЕMB 2011 a los cuales se les identificó el CHIP. Existen tres razones que pueden estar explicando estas potenciales diferencias (las cuales se pueden observar en las columnas $\mathrm{C} 1$ y C2 de la tabla 6.3, que presentan estos agregados por estrato para los dos tipos de información en la siguiente sección): 1) los agregados de la EMB 2011 representan un estimado de los hogares de Bogotá y no necesariamente reflejan el valor real, como es el caso del SUI; 2) existen 2.180 hogares de la EMB 2011 cuyo CHIP no fue posible recuperar en la base de datos SUI; 3) al observar la ЕМв 2011, existe una muestra baja de hogares en los estratos 5 y 6 , que es donde se presentan las mayores diferencias.

Este ejercicio de ajuste del cierre financiero entre las dos bases es muy importante para poder satisfacer el segundo objetivo del ejercicio que es el mantener el total de la facturación de las empresas de acueducto de Bogotá al establecer una facturación basada en la información catastral. Por tal motivo, dada la diferencia de los agregados entre el costo de prestación del servicio por estrato reportado por el sui para Bogotá y el consolidado anual por estrato a partir de la EMB 2011, se procedió a calcular un factor de ajuste que se aplica para calibrar los factores de expansión por estrato según el cociente que se presenta en la tercera columna C3 de la tabla 6.3.

\subsubsection{METOdología PARA El CÁlCULO DEL PORCENTAJE DEL GASTO TOTAL DEL HOGAR DEL PAGO EN ACUEDUCTO Y DE LAS TARIFAS DE EQUIESFUERZO BASADAS EN EL VUI}

Es importante recordar que la unidad de observación para el análisis de este ejercicio es el hogar. En este sentido, se calcula la participación del gasto en acueducto, sin subsidios ni contribuciones, sobre el gasto total del hogar, como una medida del presupuesto del hogar. En concreto, se busca medir el porcentaje que cuesta el consumo de un hogar en acueducto sobre su presupuesto, antes de la actuación del Estado a través del esquema de subsidios y contribuciones. Es esencial no olvidar que este porcentaje disminuye a medida que aumenta el ingreso del hogar, mostrando las características de 'bien necesario' del agua.

Posterior al cálculo del esfuerzo de cada hogar en agua, se establece el esfuerzo promedio para todos los hogares de la muestra como punto de referencia sobre el cual se establece el ejercicio de redistribución. Es decir, se busca encontrar el esfuerzo promedio en el servicio por parte de los hogares, 
con el fin de que cada hogar tenga esa misma participación de acueducto dentro de su estructura de gasto, independiente de su nivel de ingreso. El propósito de este equiesfuerzo es garantizar, por un lado, una redistribución significativa entre los hogares bogotanos, dado que la participación del gasto en acueducto, sin subsidios ni contribuciones, en el gasto total es decreciente el aumento con la capacidad de pago. Esto se refleja en reducciones importantes de la porción de gasto en SPD a lo largo de la distribución del ingreso. Adicionalmente, se busca que no se presenten variaciones excesivas en la nueva facturación del servicio.

El paso siguiente es poder calcular la factura mensual objetivo para cada familia; para esto, se multiplica entonces el porcentaje promedio de equiesfuerzo por el gasto total. La diferencia de este monto con el costo promedio del servicio daría el subsidio o contribución que recibiría el hogar. Este subsidio se puede normalizar por la métrica de referencia en la cantidad consumida, es decir, se puede calcular el costo implícito por unidad y la tarifa implícita de equiesfuerzo (que resultan de dividir los montos mensuales por el consumo de agua en metros cúbicos).

El último paso consiste en utilizar la información de catastro para estimar un modelo econométrico que permita imputar una tarifa 'equiesfuerzo' a partir de la información contenida en el valor unitario integral. El modelo de estimación propuesto es establecer una regresión lineal simple cuya variable resultado, o por explicar, es la tarifa implícita 'equiesfuerzo' calculada en los pasos anteriores y la variable exógena, o explicativa, es el promedio por decil de gasto total del vUI. Esta regresión se puede ver expresada en el siguiente modelo lineal:

$$
\text { TarifadeEquiesfuerzo }=\beta_{0}+\beta_{1} * V U 1+\varepsilon_{i}
$$

Después de implementar este modelo, se recuperan los parámetros estimados para poder imputar la tarifa equiesfuerzo, dado el valor de avalúo vUi que le corresponde a cada hogar, y, de este modo, se determina la tarifa unitaria estimada:

$$
\text { Tarifade } \widehat{\text { Equiesfuerzo }}=\widehat{\beta}_{0}+\widehat{\beta}_{1} * \text { VU1 }+\varepsilon_{i}
$$




\subsection{Caso de uso de clasificación sin estratos en acueducto: resultados}

Los resultados del ejercicio se presentan en las tablas 6.2 en adelante, en donde se muestran varios de los resultados, los cuales se resumen por decil de gasto. La tabla 6.2 muestra el esfuerzo promedio, o participación del gasto en el servicio como porcentaje del gasto total, por decil de capacidad de pago corriente según estrato y los respectivos marginales que realizan los hogares para el pago del servicio de acueducto (sin subsidios ni contribuciones).

El promedio global para Bogotá de la participación del gasto en acueducto sobre el gasto total es del 1,10\%. De la información agrupada en la tabla 6.2, se obtiene una implicación lógica inmediata en donde las familias con baja capacidad de pago, medida por el ICPc, destinan una mayor proporción de su presupuesto a pagar el servicio de acueducto, a diferencia de aquellos hogares caracterizados con una mayor capacidad de pago. Adicionalmente, se puede observar que algunos hogares con baja capacidad de pago y que están habitando viviendas de estrato 4 , es decir, el estrato neutro, donde no pueden ser beneficiadas por subsidio alguno, se encuentran haciendo un esfuerzo significativo en el pago del servicio de acueducto.

En concreto, se pueden destacar dos aspectos generales de la información agrupada por la incidencia marginal entre decil de la capacidad de pago y el estrato. Primero, a medida que el decil de capacidad de pago o que el estrato socioeconómico aumenta, disminuye el esfuerzo que harían los hogares por el servicio de acueducto, pasando del 4,83\% para el primer decil al 0,51\% para el último decil, y, de manera similar, se obtiene una participación del gasto en acueducto del 3,18\% en el estrato 1 y del 0,57\% en el estrato 6. Segundo, al observar las diferencias por decil del ICPc diferenciado por estrato, el porcentaje de gasto es bastante heterogéneo. Para los tres primeros deciles, la proporción de gasto se incrementa a medida que aumenta el estrato (hasta el estrato 3), lo cual es el resultado de que hogares pobres clasificados en estratos mayores deben realizar más esfuerzo al no ser beneficiarios de los subsidios cruzados atados a la estratificación. Para los demás deciles, el esfuerzo tiende a caer (con algunas excepciones) a medida que aumenta el estrato. Como se expuso en la metodología de cálculo de las facturas 'equiesfuerzo', se utiliza el promedio del porcentaje del gasto en acueducto sobre el gasto total de todos los hogares de Bogotá, el cual es del 1,103\%. Posteriormente, se multiplica 
este porcentaje por el gasto total de cada hogar y se obtienen los valores de factura promedio 'equiesfuerzo'.

Tabla 6.2. Promedio del esfuerzo en acueducto por estrato según decil de capacidad de pago corriente

\begin{tabular}{c|c|c|c|c|c|c|c}
\hline $\begin{array}{c}\text { Decil ICPc/estrato } \\
\text { según EMB 2011 }\end{array}$ & 1 & 2 & 3 & 4 & 5 & 6 & Total \\
\hline 1 & 4,04 & 5,05 & 5,65 & 3,06 & & & 4,83 \\
\hline 2 & 3,58 & 3,64 & 4,19 & 2,76 & & & 3,72 \\
\hline 3 & 2,56 & 2,73 & 3,19 & 2,92 & & & 2,83 \\
\hline 4 & 2,32 & 2,28 & 3,17 & 1,52 & & & 2,6 \\
\hline 5 & 2,16 & 2,18 & 2,01 & 1,56 & & & 2,1 \\
\hline 6 & 2,13 & 2,01 & 1,61 & 1,34 & 1,08 & & 1,77 \\
\hline 7 & 1,88 & 1,58 & 1,71 & 1,12 & 0,99 & & 1,61 \\
\hline 8 & 1,26 & 1,45 & 1,09 & 0,85 & 0,94 & 2,09 & 1,11 \\
\hline 9 & 0,98 & 1,16 & 0,74 & 0,71 & 0,66 & 0,59 & 0,76 \\
\hline 10 & 0,9 & 0,69 & 0,48 & 0,46 & 0,58 & 0,53 & 0,51 \\
\hline Total & 3,18 & 2,84 & 1,91 & 0,73 & 0,66 & 0,57 & 1,1 \\
\hline
\end{tabular}

Fuente: elaboración de los autores con base en la EMB 2011 y SUI.

Del proceso de emparejamiento de las bases, se realizó una descripción de los montos que se obtienen, por un lado, de la ESP y, por el otro, de la EMB 2011. Los montos agregados del consumo en acueducto se resumen en la tabla 6.3 y se discriminan por estratos. Como se puede observar, el costo de prestación de las ESP ${ }^{11}$ de acueducto en Bogotá difiere del que se obtiene una vez homologada la información de las dos bases con que se está trabajando. La columna 1 evidencia los valores calculados por la ESP; la columna 2, los valores de consumo obtenidos a través de la base de hogares de la EMB 2011, donde se identificó el CHIP. La columna 3 presenta la razón entre los valores en la columna 1 y la columna $2(\mathrm{C} 1 / \mathrm{C} 2)$ y, por lo tanto, esto constituye los factores de ajuste para lograr una armonización de la base con el referente reportado por la ESP al SUI y obtener los mismos costos de prestación oficiales.

11 Además de la Empresa de Acueducto de Bogotá, hay otros prestadores del servicio de acueducto. 
El monto global de las facturas equiesfuerzo se exponen en la columna C4 y su diferencia con el costo total de prestación del servicio se muestra en la columna C5, y resulta en el balance de los subsidios y contribuciones por estrato. En agregado, los hogares que hoy están en estrato 1, 2 y 3 muestran un balance negativo (pago-costo), mientras que aquellos que hoy se encuentran en estratos 4 al 6 presentan un balance positivo. Esta diferencia, sin embargo, no se puede entender como que los hogares de los dos primeros estratos actuales serán los únicos subsidiables. La comparación por estrato recoge, por un lado, la variación efectiva de la tarifa, pero, por otro, la reclasificación por errores de inclusión.

Tabla 6.3. Costo de prestación (pesos), facturación por consumo por estrato (anual) y factor de ajuste

\begin{tabular}{c|c|c|c|c|c}
\hline Estrato & $\begin{array}{c}\text { Costo de } \\
\text { prestación ESP } \\
(\mathrm{C} 1)\end{array}$ & $\begin{array}{c}\text { Facturación sin subsidios } \\
\text { ni contribuciones EMB } \\
2011 \text { y suI (C2) }\end{array}$ & $\begin{array}{c}\text { Factor de } \\
\text { ajuste (C1/ } / \\
\text { C2) }\end{array}$ & $\begin{array}{c}\text { Facturación por con- } \\
\text { sumo con la propuesta } \\
\text { de 'equiesfuerzo' (C4) }\end{array}$ & C5 = C1 - C4 \\
\hline 1 & $\$ 6.112 .181 .968$ & $\$ 4.980 .214 .225$ & 1,22 & $\$ 2.671 .854 .760$ & $\$ 3.440 .327 .208$ \\
\hline 2 & $\$ 28.878 .374 .586$ & $\$ 28.321 .184 .693$ & 1,02 & $\$ 15.113 .476 .002$ & $\$ 13.764 .898 .584$ \\
\hline 3 & $\$ 30.038 .570 .284$ & $\$ 26.638 .458 .521$ & 1,13 & $\$ 26.332 .688 .084$ & $\$ 3.705 .882 .200$ \\
\hline 4 & $\$ 12.801 .689 .337$ & $\$ 6.968 .847 .370$ & 1,84 & $\$ 23.426 .948 .345$ & $\$-10.625 .259 .008$ \\
\hline 5 & $\$ 4.787 .739 .924$ & $\$ 2.567 .921 .340$ & 1,86 & $\$ 9.209 .659 .622$ & $\$-4.421 .919 .698$ \\
\hline 6 & $\$ 4.968 .383 .552$ & $\$ 2.215 .822 .173$ & 2,24 & $\$ 10.832 .312 .838$ & $\$-5.863 .929 .286$ \\
\hline Total & $\$ 87.586 .939 .650$ & $\$ 87.624 .396 .830$ & na & $\$ 87.586 .939 .651$ & \\
\hline
\end{tabular}

Fuente: elaboración de los autores con base en la EMB 2011 y suI.

La tabla 6.4 presenta las principales estimaciones. Las dos últimas columnas contienen el costo unitario implícito de prestación del servicio de acueducto y la tarifa implícita de equiesfuerzo. La diferencia de estas dos columnas es el subsidio y contribución por consumo de metro cúbico de agua. En este sentido, vemos que, mientras el costo medio es constante entre deciles, pues no incluye los subsidios ni las contribuciones, la tarifa media implícita es creciente a medida que la capacidad de pago aumenta, de acuerdo con el propósito definido. Si todos los hogares bogotanos realizaran en promedio un esfuerzo similar, manteniendo el consumo fijo, los deciles 1 al 7 pagarían una tarifa mayor al costo, es decir, recibirían un subsidio, mientras que aquellos del decil 8 en adelante tendrían que pagar una contribución. 


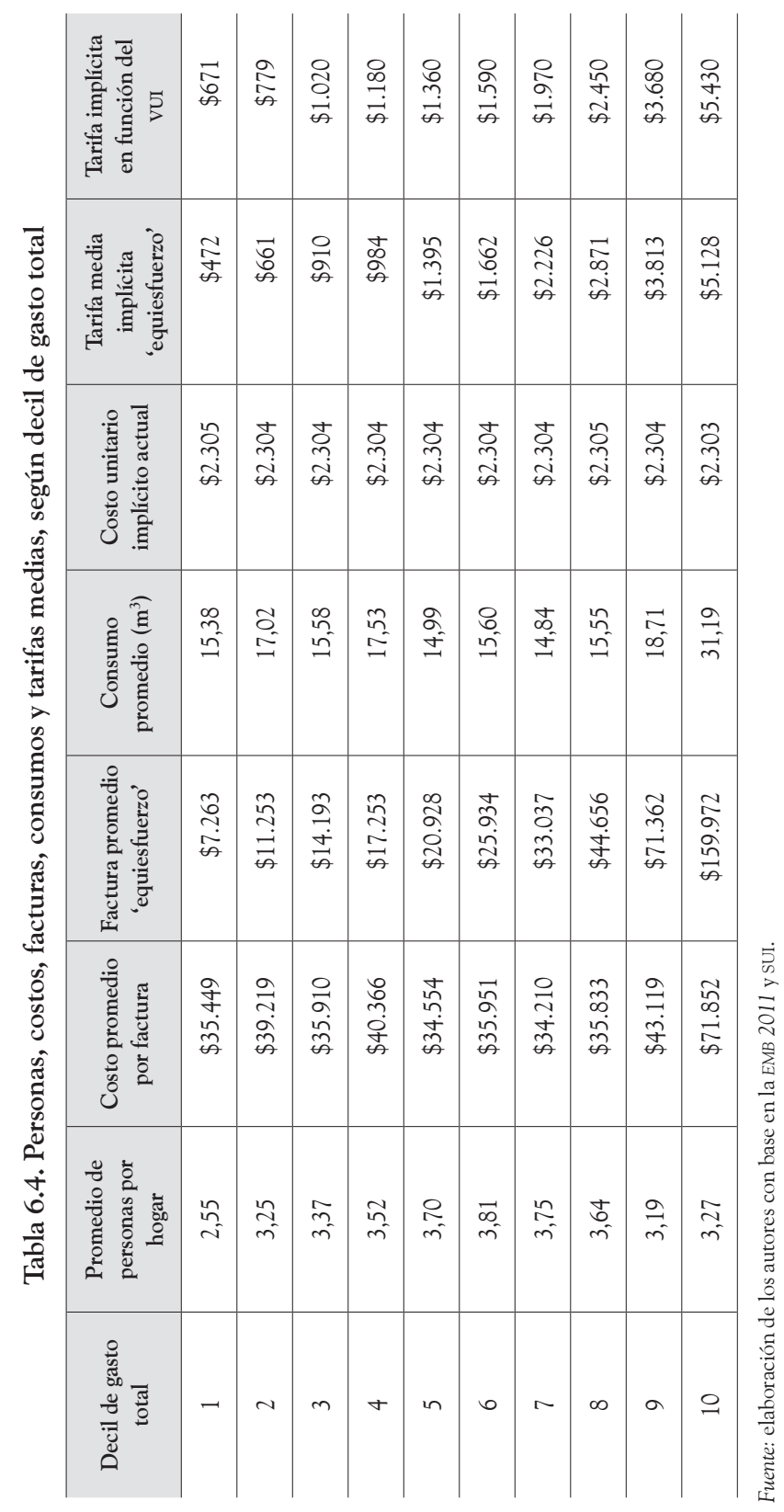




\subsubsection{MODELO DE TARIFAS EN FUNCIÓN DEL VALOR UNITARIO INTEGRAL}

Para definir el cobro final por unidad de consumo teniendo en cuenta el avalúo, se estima el modelo de regresión lineal simple de las tarifas implícitas equiesfuerzo en función del valor unitario integral (VUI). La estimación del modelo dio como resultado:

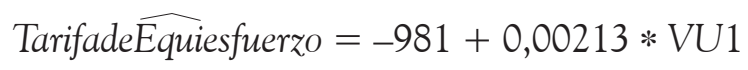

$(177,6) \quad(0,00013)$

La regresión indica que, por cada 100 mil pesos de vUI, se aumenta el cobro por metro cúbico en 243 pesos. Con la estimación obtenida, se procede a asignar el valor de la tarifa para el servicio de acueducto en cada uno de los hogares de la EMB 2011 y se multiplica este valor por el consumo del hogar para obtener el valor de la factura que debería cancelar cada hogar. La última columna de la tabla 6.4 presenta el valor por facturación como función del valor unitario integral, así como su respectiva tarifa implícita. El modelo guarda similitud con las tarifas y las facturas equiesfuerzo, como era de esperarse por construcción.

En el transcurso del capítulo, se ha discutido acerca del esfuerzo que hacen los hogares para el pago del servicio de acueducto en Bogotá, D.C.; la figura 6.2 ilustra los resultados principales por decil de capacidad de pago. Las líneas continuas representan el costo promedio del servicio y el esfuerzo que los hogares harían sin tener subsidio, mostrando la naturaleza del servicio y su fuerte regresividad en caso de no tener un sistema cruzado de subsidios. El esfuerzo que realizarían los hogares para el pago de acueducto inicia con un $6,16 \%$ para el primer decil y disminuiría considerablemente hasta el 0,87\% (alta pendiente negativa) para el último decil. Las líneas discontinuas, por su parte, presentan el panorama de los hogares una vez definida la tarifa de equiesfuerzo. La línea intermitente naranja muestra el objetivo de que cada hogar termine haciendo el mismo esfuerzo, mientras que la azul expone el resultado de la tarifa por metro cúbico que pagaría cada familia, que termina en un esquema progresivo en el cobro unitario e igual en el esfuerzo. 
Al comparar las dos líneas azules, tenemos la diferencia entre el cobro de tarifa y el costo medio de cada decil. Así, son los hogares entre el decil 1 y 7 los que recibirán un subsidio implícito en su cobro, mientras que aquellos entre el 8 y el 10 pagarían una contribución.

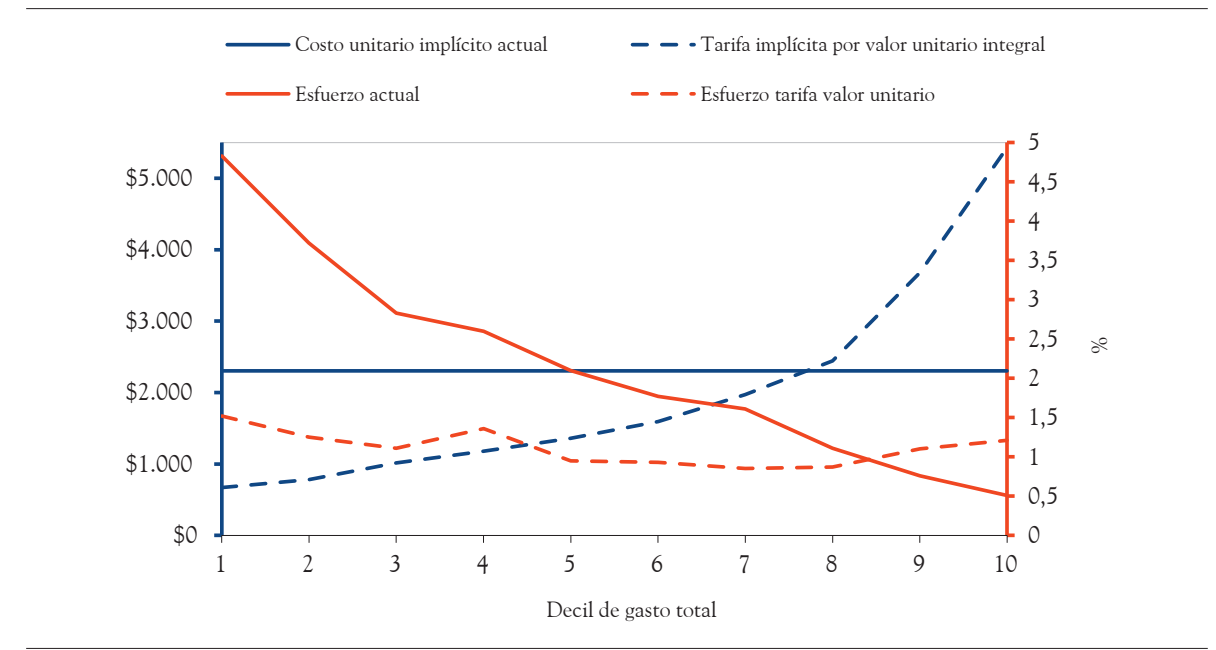

Figura 6.2. Tarifas implícitas y esfuerzo de los hogares

Fuente: elaboración de los autores con base en la EMB 2011 y SUI.

\subsubsection{IMPACTO EN LOS HOGARES}

Para entender el efecto de la propuesta en los hogares, debe compararse el cobro final equiesfuerzo con el escenario en que se enfrentan actualmente, es decir, el pago presente (con subsidios y contribuciones). La tabla 6.5 muestra por decil la proporción de hogares que les disminuye o les aumenta el pago por unidad. En todos los deciles, hay hogares que percibirían un aumento o una disminución de tarifas. Sin embargo, el resultado evidencia que, a medida que la capacidad de pago aumenta, es mayor el porcentaje de hogares que perciben un incremento de tarifa.

Por último, la tabla 6.6 presenta la diferencia promedio entre el pago actual y el pago de la nueva propuesta. Así, a pesar de que en promedio el subsidio implícito de la tarifa lo recibirían hogares en deciles 1 al 7, solo aquellos en los dos últimos tendrían un incremento en su pago mensual. Se tiene que, en promedio, el pago disminuye más de $\$ 10.000$ respecto a la facturación total actual en los deciles del 1 a 6 ; una disminución de menos de $\$ 5.000$ para los deciles 7 y 8 ; y un aumento en los deciles 9 y 10. 
Tabla 6.5. Proporción de hogares según el tipo de cambio en la tarifa

\begin{tabular}{c|c|c}
\hline Decil del gasto total & $\begin{array}{c}\text { Porcentaje de hogares en los que la tarifa } \\
\text { implícita actual disminuye }\end{array}$ & $\begin{array}{c}\text { Porcentaje de hogares en los que la tarifa } \\
\text { implícita actual aumenta o se mantiene }\end{array}$ \\
\hline 1 & $95,2 \%$ & $4,8 \%$ \\
\hline 2 & $92,6 \%$ & $7,43 \%$ \\
\hline 3 & $90,9 \%$ & $9,09 \%$ \\
\hline 5 & $85,9 \%$ & $14,14 \%$ \\
\hline 6 & $82 \%$ & $17,9 \%$ \\
\hline 7 & $77,7 \%$ & $22,3 \%$ \\
\hline 9 & $69 \%$ & $31 \%$ \\
\hline 10 & $59 \%$ & $40,9 \%$ \\
\hline
\end{tabular}

Fuente: elaboración de los autores con base en la EMB 2011 y sUI.

Tabla 6.6. Comparación de promedios de facturación actual frente a la facturación en función del valor unitario integral

\begin{tabular}{c|c|c}
\hline Decil de gasto total & $\begin{array}{c}\text { Promedio de facturación actual mes } \\
\text { (-subsidios }+ \text { contribuciones })\end{array}$ & Promedio de facturación en función del vUI \\
\hline 1 & $\$ 24.380$ & $\$ 10.000$ \\
\hline 2 & $\$ 27.630$ & $\$ 12.760$ \\
\hline 3 & $\$ 27.260$ & $\$ 14.280$ \\
\hline 5 & $\$ 33.020$ & $\$ 21.960$ \\
\hline 6 & $\$ 28.690$ & $\$ 17.960$ \\
\hline 7 & $\$ 31.240$ & $\$ 21.850$ \\
\hline 8 & $\$ 32.950$ & $\$ 35.540$ \\
\hline 10 & $\$ 38.296$ & $\$ 71.630$ \\
\hline
\end{tabular}

Fuente: elaboración de los autores con base en la EMB 2011 y suI.

\section{Conclusiones}

La estratificación como instrumento para focalizar subsidios a los SPD se vinculó desde un principio a objetivos como aumento de la cobertura, solidaridad, 
aspectos redistributivos o de disminución de la pobreza, y a la sostenibilidad financiera y fiscal del esquema. Es evidente que la política estatal desarrollada a partir de la Ley 142 de 1994, anclada en la estratificación socioeconómica, permitió mantener avances considerables en la cobertura de acceso de los hogares colombianos a los servicios públicos básicos. Este es tal vez el logro más importante de esa política social, si bien a nivel rural es aún mucho lo que puede hacerse. Sin embargo, como instrumento para reducir la desigualdad del ingreso, los logros son muy magros. A nivel del hogar promedio de los estratos 1 y 2 de menores ingresos, es cierto que la política de subsidios cruzados mantenida y desarrollada por la Ley 142 ha sido exitosa en liberar capacidad de gasto (ver Gallego, Gutiérrez, Ramírez y Sepúlveda, 2014). No obstante, visto a un nivel más global, el impacto de esa política no ha generado movimientos importantes hacia una mejor distribución del ingreso. Ello ha sido el resultado de los errores de inclusión que, por su misma naturaleza, son regresivos en el ingreso y de los errores de exclusión, que generan efectos directos de regresividad al no permitir a hogares de bajos ingresos gozar de los subsidios. Por último, no se ha podido, desde el mismo inicio del esquema, mejorar la factibilidad y sostenibilidad fiscal del sistema dados esos errores de inclusión.

Este capítulo presenta un primer ejercicio exploratorio que permite la definición de subsidios y contribuciones, ligadas a la información catastral (valor unitario integral por metro cuadrado), sin definir estratos socioeconómicos. Basados en la alta correlación que tiene la capacidad de pago con variables catastrales como el avalúo, el ejercicio establece el pago por unidad de consumo de agua, de manera que cada hogar en Bogotá destine una misma proporción de su gasto total al gasto en el servicio de acueducto. Adicionalmente, garantiza que el total de pagos cubran los costos de provisión del servicio. Se supone que el consumo actual de las familias no se altera frente al cambio propuesto. Ejercicios futuros deben incorporar el cambio en el consumo de agua teniendo en cuenta la elasticidad precio de la demanda.

Teniendo como objetivo que el esfuerzo de gasto en agua sería el promedio del esfuerzo actual de todos los hogares en Bogotá, y mantener los ingresos por facturación de las EPS, el ejercicio muestra que se tendría un subsidio implícito en la factura para hogares de los primeros siete deciles de capacidad de pago y contribuciones implícitas para los últimos tres deciles. 
De manera sencilla, el resultado ilustra cómo un desmonte de la estratificación puede llegar a un esquema directo y simple, de forma que se permite continuar con subsidios cruzados para SPD, sin traumatismos significativos para los hogares bogotanos, que, además, podrían ser aligerados con una prudente transición. En promedio, el cambio de esquema llevaría a una disminución del pago en acueducto para familias pertenecientes a los primeros ocho deciles de capacidad de pago del Distrito Capital, y a un aumento, en promedio, de los hogares en los últimos dos deciles. Para una propuesta final, sin embargo, debe tenerse en cuenta la sensibilidad en el consumo de los hogares ante el cambio de tarifas, y avanzar en un esquema de tarifas diferenciadas, de modo que el sistema cruzado no resulte en un subsidio a los consumos totales de servicios públicos, sino que garantice que estos subsidios se focalicen en el pago de consumos básicos de subsistencia.

A futuro, se puede considerar la construcción de registros de hogares que permitan una focalización más directa frente a la capacidad de gasto de cada familia o que sea complementaria al esquema base que utilizarían las comisiones de regulación en la definición de los esquemas tarifarios. Una alternativa es el uso del SISBEN, que actualmente es mecanismo de focalización para el otorgamiento de diferentes subsidios sociales. No obstante, también presenta limitaciones importantes. Por un lado, pueden darse comportamientos estratégicos de parte de los hogares en los procesos de actualización del SISBEN que los lleva a pasar de no ser beneficiarios a serlo, con los consecuentes costos fiscales y efectos regresivos sobre la distribución del ingreso. La experiencia internacional sobre el uso de mecanismos de focalización sobre las personas y no sobre los predios (o áreas y zonas geográficas) exige la existencia de mecanismos directos de (auto)identificación que, por su misma naturaleza, son dinámicos, más igualitarios y menos sujetos a irregularidades administrativas. En el país, aún no se cuenta con un marco institucional y de sistemas de información que permita usar mecanismos de focalización más directos sobre las condiciones de vida de los individuos, sin dejar de resaltar que los registros existentes, al no ser universales, no permiten un sistema cruzado de subsidios como el actual. El uso de la información disponible que tiene Bogotá en sistemas como el catastral no puede pasarse por alto en la intención de una mejora de las herramientas de focalización y redistribución de ingresos. 